\title{
AN ARCHITECTURE FOR PROVIDING RANGE EXTENSION BY DEPLOYING MOBILE GATEWAYS IN AD HOC NETWORKS
}

\author{
Mohiuddin Ahmed ${ }^{1}$, Srikanth Krishnamurthy ${ }^{2}$, Randy Katz ${ }^{3}$, and Son Dao ${ }^{4}$ \\ 1,4 Information Sciences Laboratory, HRL Laboratories, LLC, Malibu, CA, \{mohin,skdao\}@wins.hrl.com \\ ${ }^{2}$ Computer Science Department, University of California, Riverside, CA, krish@cs.ucr.edu \\ ${ }^{3}$ Computer Science Department, University of California, Berkeley, CA, randy@ cs.berkeley.edu
}

\begin{abstract}
The dynamic nature of a mobile ad hoc network (MANET) may result in a cluster of nodes being isolated from the rest of the network, especially when deployed in a terrain with blockages. To provide connectivity between the partitions of an ad hoc network that might occur due to mobility, a 'range extension' network can be employed. Such a network might consist of airborne communication platforms, or geostationary/low-earth-orbit satellites maintaining communication links with specific 'gateway' nodes that are dispersed among the mobile ground nodes. Thus, to communicate with a node that is geographically distant or belongs to a different network partition, an ad hoc node can relay its data packets through an appropriate mobile gateway and via the range extension network.

In such an architecture, MANET is divided into different domains with a mobile gateway deployed for each domain. The objective, then, is to determine the position and trajectory of the gateways to optimize network performance metrics such as throughput and latency. In this paper, computation of the optimal position for a gateway is shown to be equivalent to a linear optimization problem by means of some simplifying but realistic assumptions. An algorithm is proposed for the control of the gateway trajectory. The practical constraints imposed by the velocity and maneuverability of the gateways are taken into account. Simulation results show a 10-15\% improvement in the throughput and latency, per gateway domain, if a gateway has a dynamic trajectory whose locus follows the computed optimal position, as compared to a gateway that is statically placed at a fixed position, or to a gateway that has a random trajectory.
\end{abstract}

Keywords - mobile ad hoc networks, gateway, convex optimization, trajectory control.

\section{INTRODUCTION}

$\mathbf{M}$ OBILE ad hoc networks are multi-hop packet networks that have no centralized or pre-determined network architecture [1]. Instead, the nodes of the network cooperate in a distributed fashion to build a dynamic network infrastructure. This flexiblity makes ad hoc networks appropriate for many applications such as: connecting mobile computers in an office or home environment, deploying wireless sensors in remote or inhospitable terrain, coordinating disaster relief efforts after natural catastrophes, or in tactical deployments for situation awareness applications [2]. However, this lack of centralized organization creates challenges for achieving network scalability. Furthermore, due to the range limitations of ad hoc nodes, the network might often be geographically divided into isolated partitions. In order to achieve scalability in terms of efficient communications between geographically distant nodes or between nodes that belong to different isolated partitions (each of which is an ad hoc group by itself), it is desirable to provide a minimal supporting infrastructure in the form of a range extension network. This infrastructure is also essential to interface the MANET with the Internet.

We envision the range extension network to rely on airborne or satellite relay nodes in order to interconnect the isolated partitions of an ad hoc network. However, this will require the deployment of special nodes on the ground, among the ad hoc network nodes, that are equipped with the appropriate hardware for communicating with the satellite/airborne nodes. This hierarchical routing architecture, therefore, can be visualized to consist of a range extension network consisting of satellites or airborne nodes and mobile gateways that provide the interface for the communications between nodes in different domains and/or to a backbone network (Figure 1). Similar architectures have previously been considered for enabling hierarchical routing or multicasting ([4]-[6]). In contrast, we consider the question that arises as to where a gateway that is affiliated with an ad hoc team of mobile units ought to be located, relative to these other mobile units. We design a methodology for defining the gateway trajectory based upon the location, loading, etc. of the other nodes in the ad hoc group that the gateway serves. We show that network performance improves (for communications involving nodes in different clusters or teams), in terms of throughput and latency, if the gateway trajectory is computed based on this methodology. Thus, in our scheme, the mobile gateways react and alter positions in an attempt to maximize the efficiency of inter-domain communications, in addition to providing a means of range extension.

We derive a relatively simple analytical formulation for the optimal gateway position. This formulation reduces the problem to a linear optimization problem. This is discussed in Section III. We also provide an algorithmic implementation of the formulation in this section, and discuss the effects of 
some of the system parameters on the performance. In Section IV, we estimate the overhead and the computational complexity incurred in implementing this architecture with the aid of typically used media access control (MAC) and routing protocols. In Section V, we discuss our simulation framework and some results.

\section{Network ARChitecture}

The ad hoc network architecture may be envisioned to contain groups of mobile ad hoc nodes operating in a deployment area with restrictions (e.g. troop divisions in a mountainous area or a region where certain nodes cannot reside, such as hazard zones, regions of radio obscurity, etc. These restricted zones are collectively referred to as blockages). Each group would have one or more gateways capable of communicating with an airborne or satellite node with which it has a direct line-of-sight connection, e. g. as shown in Figure 1. The gateway in each domain is then the conduit via which the ad hoc nodes in separate domains can send data packets to each other, or to a wired infrastructure, with the routing assistance of airborne nodes.

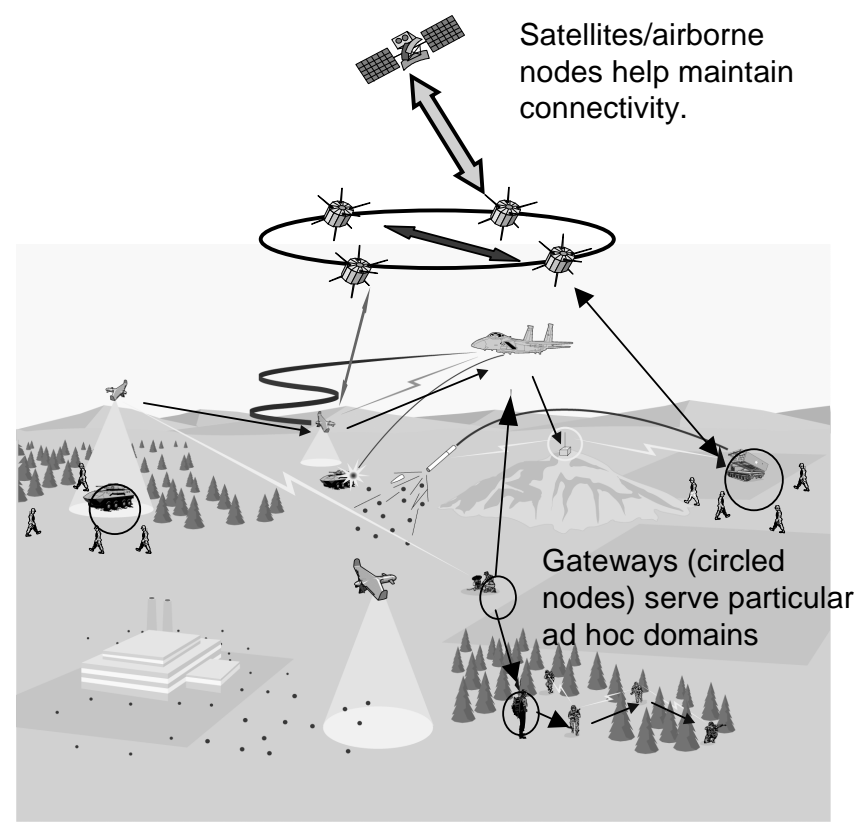

Fig. 1. Ad hoc network of groups of mobile nodes and gateways.

Since the nodes forming a particular group are mobile, the objective is then is to determine the 'optimal' trajectory of the gateway associated with that group. For communication intended for nodes within a given group, the nodes would not be compelled to use the gateway, but would instead rely on the underlying MANET architecture using traditional routing, MAC protocols, etc. By intelligently positioning the gateway, we might expect to achieve better network performance for inter-domain node communications, (i.e. data communication between nodes that are in the domains of different, possibly geographically isolated gateways) than if the gateway were allowed to move randomly with respect to the nodes it its domain. The performance metrics that could potentially be improved include: inter-domain network data throughput; inter-domain network packet transport delay; total power expended; data transmission reliability (packet drop/error rate) and the volume of the network control messages and resulting signaling overhead, among others.

It is shown in the next section that the gateway positioning methodology can be formulated and solved as a simplified linear optimization problem. The technique essentially corresponds to a weighted centroid computation for the gateway position, as the nodes in its group move about the domain.

The details of the actual communication mechanisms that enable the MANET to function are not directly relevant in the development of our analytical formulation for computing the optimum trajectory that our gateway ought to follow (Section III). For intra-domain node communications, the MANET could rely on well-established protocols such as the IEEE 802.11 MAC protocol for media access control [7], DSR, DSDV, or AODV for routing [3], etc. to establish and maintain connectivity. For inter-domain node communications, data will have to be routed through the gateway and via the range extension network.

\section{Gateway Trajectory Update Algorithm: FORMULATION AND ANALYSIS}

We now describe the algorithm for determining the trajectory of the mobile gateways such that it is optimal in terms of its 'relative position' with respect to the group of ad hoc nodes that it serves. We describe our algorithm assuming that there is a single gateway per domain. However, it is possible for several nodes among a cluster of nodes to be able to communicate with the range extension network and hence, any of these nodes could assume the role of a gateway. Fortunately, in all these cases, the base algorithm that we propose remains unchanged. Increased layers of complexity can be added to the base algorithm to enable the gateways to participate in node 'hand-off' as in cellular networks, or to intelligently share the load generated by the nodes in the shared domains. These features are not discussed further in this paper due to space limitations.

Intuitively, it can be argued that the gateway should always be positioned closest to the weighted geographic centroid of the node positions in the domain. This is essentially what our algorithm does. The weighting factors are the parameters that the gateway can take into account during the optimization process (e.g. node positions, each node's offered load, data traffic patterns, priority of the generated traffic, the channel signal to interference noise ratio (SIR), among others). The relative importance of the parameters are determined by the specific network metrics of interest (mentioned earlier in Section II). For the purposes of this discussion, we consider po- 
sition and offered load as the primary parameters and the network throughput and the average delay experienced by interdomain data packets as the basic performance metrics. Thus, we make the following assumptions about the nodes in the network:

1) Each node is equipped with a GPS device that enables the node to determine its position.

2) Each node can estimate its offered load in real time.

3) Terrain information (such as specific coordinates or boundaries of the domain, radio null regions, etc.) is available at each gateway. This can easily be made available at network inception. (Position based schemes have previously been suggested and studied for ad hoc networks, [4], [6]).

Furthermore, the domain and the terrain blockages can always be bounded by simple rectangular regions where the granularity can chosen according to the desired level of resolution. This representation is useful since the constraints that govern the position of a gateway can then be described by simple linear equations (based on the coordinates of the rectangular boundaries), rather than by complex non-linear expressons.

Since MANETs are multi-hop networks, nodes outside the single-hop radio range of the gateway and will have to route their data packets to the gateway via multiple hops through other ad hoc nodes. However, the cost function that we use in computing the weighted geographic centroid takes the offered load of the individual nodes and the priorities of the packets generated at each node into account. Thus, with successive iterations of the trajectory control algorithm, the gateway eventually will be closest to the most heavily loaded (or highest priority) nodes, which can thus reach the gateway in a single, or minimum number of hops.

\section{A. Optimization Formulation}

For a domain with $n$ nodes, the optimization problem can be formulated as:

$$
\begin{array}{cc}
\operatorname{minimize} & \sum_{i=1}^{n} \eta\left(\tau_{i}, \rho_{i}\right) \cdot\left|\mathbf{x}_{0}-\mathbf{x}_{i}\right| \\
\text { subject to } & \mathbf{w}_{1} \leq \mathbf{x}_{0} \leq \mathbf{w}_{2} \\
\mathbf{x}_{0} & \geq \mathbf{b}_{2 k} \\
\mathbf{x}_{0} & \leq \mathbf{b}_{1 k}
\end{array}
$$

The optimization variable $\mathbf{x}_{0}$ in the norm minimization expression, Eq. 1, is the desired gateway position, represented by a 2-D position vector with reference to any suitable origin in the terrain of interest. The other $\mathbf{x}_{i}$ 's represent the coordinate vectors of the mobile nodes and are obtained at each previously defined sampling instant. The weighting factor, $\eta\left(\rho_{i}, \tau_{i}\right)$ is a user defined function that depends on the $i^{t h}$ node's load, $\rho_{i}$, and priority, $\tau_{i}$. We do not consider the data load due to intra-domain communication among the nodes, although this may affect the available bandwidth for interdomain communications. Depending on the type of traffic being generated by the nodes, the function $\eta\left(\rho_{i}, \tau_{i}\right)$ can be defined appropriately to reflect CBR, or variable bit rate (VBR) traffic and with or without defined priorities. The terms $\mathbf{w}_{i}$ are the vector coordinates representing the rectangles circumscribing the domain (bottom left and the top right points), and $\mathbf{b}_{i k}$ 's are similar vectors that represent the boundary of the $k^{t h}$ blockage. Therefore, we are minimizing the sum of the weighted geometric distance from the gateway to each of the nodes, subject to linear boundary and blockage constraints.

This problem is a non-linear optimization problem. However, since the cost function is the $L^{2}$ norm and the constraints are linear, the problem is a convex program that can be transformed into a simple linear program (LP), which can then be efficiently solved via modern numerical interior point methods [10]. Thus, the Gateway Trajectory Update Algorithm can be represented as shown below, and the gateway would execute this as a continuous loop with an appropriate looping interval.

- Input constants (set 'a priori'): terrain and blockage boundaries, sampling times, optimization metric of interest.

- Output: optimum gateway location computed at specific sampling times.

- $\{$ While nodes in the domain have inter-domain data packets to send\}, DO:

1) Collect or estimate the position of each node, $\mathbf{x}_{i}$, at each sampling instant.

2) Collect from each node, an estimate of its current load and the priority that it desires.

3) Perform a local computation to solve the LP equivalent to the optimization problem in Equation 1 and obtain optimum gateway location for that sampling instant.

4) Move towards the optimal location in the most suitable manner, as allowed by the physical constraints (The problem of navigating from one location to another, on a 2-dimensional surface with obstacles with no preestablished paths, is a vast area of research in robotics and is not discussed in this paper [13]).

5) Repeat at next sampling instant.

The motion of the gateway can be further governed by certain rules to prevent race conditions. As an example, one can have a hysteresis rule that helps prevent excessive gateway sensitivity, wherein a computed 'new' gateway location has be greater than some minimum pre-specified $\delta$ units from the present location before we decide to move the gateway.

\section{Computational Complexity}

\section{A. MAC Protocol, Routing Support and Overhead}

The overhead incurred in the execution of the trajectory control algorithm can be estimated as follows. In steps 1 and 2 of the algorithm, the gateway has to obtain state information from all the nodes. This can be pre-configured at network deployment, or can acquired on a reactive basis, in which case it is about the same as discovering a specific node within a MANET. However, to obtain position/loading information, if there are $n$ mobile nodes in the network, then for these nodes to transmit an update every sampling period, the 
number of messages required is of the order of $O\left(n^{2}\right)$ (assuming flooding is employed to transport these messages) [5]. We note, however, that this control information can simply be 'piggy-backed' onto the routing update messages (most of these parameters are usually 8-bit or 16-bit numbers, per node, so payload data length is not an issue), or embedded in the MAC layer 'hello' messages, or even piggy-backed onto the data payload that is routed to the gateway. This makes the overhead required for gathering state information for the algorithm to be on the same order as that required for the operation of the underlying routing and MAC protocols (routing protocols are discussed in [3]).

\section{B. Optimization Complexity}

The computation of the trajectory itself involves solving a linear program numerically. It is well known that modern interior point LP solvers have a worst-case performance of $O\left(n^{3}\right)$ [10], where $n$ is the number of variables in the LP. Thus, for a network of 100 nodes, each of which is assumed to generate inter-domain data packets, we would expect calculations on the order of $100^{3}$ or 1 million iterations per update period. A typical update period is of the order of 0.5 seconds in our simulations. Currently available 'off-the-shelf', inexpensive microprocessors can process on the order of tens of millions of instructions per second [14]. For a network with 1000 nodes, the complexity increases significantly, requiring 1 billion iterations per update period. In such cases, however, it is far more efficient to simply deploy more gateways, and thus sub-divide the larger domain into smaller domains of ad hoc networks.

\section{Simulation Framework AND RESUlts}

To evaluate the algorithm, the $n s-2$ network simulator, release $2.1 \mathrm{~b} 6$ [12] was our primary simulation platform, with code extensions for the 802.11 MAC and DSDV routing [9], as implemented by Carnegie Mellon University for wireless ad hoc networks. We also conducted simulations with appropriate modifications to the Dynamic Source Routing (DSR) algorithm [11]. Our results were similar and we omit a discussion of this due to space limitations. The LP algorithm itself was integrated into the $n s-2$ simulation framework by means of a $\mathrm{C}$ function call from the main program code. To implement the optimization algorithm, we used a modified version of PCx [15]. We were interested only in the inter-domain networking performance and consequently, all the data packets that the nodes generated in each domain were always deterministically addressed to the gateway. Intra-domain traffic in the MANET does not affect the gateway trajectory. The range extension network beyond the domain of the gateway was itself considered to be a 'black-box'.

The first case that we consider is that of equally loaded nodes generating packets of the same priority. In this case, $\eta\left(\rho_{i}, \tau_{i}\right)=1$, for all $i$, and the problem is now equivalent to minimizing the sum of the distances from the gateway to the nodes, subject to the boundary and blockage constraints.

The results shown in Figure 2 through Figure 4 are for the following system parameters: a rectangular area of size 10,000 units by 10,000 units; a network of mobile ad hoc nodes ranging from 10 to 100 per domain; nodes assigned random velocities chosen uniformly between 0 units/s (stationary) and 25 units/s, moving in accordance with the random waypoint model. The gateway velocity is chosen to be at most one and a half times the maximum speed of the ad hoc nodes. All the nodes are equally loaded and generate traffic $50 \%$ of the time. The mobile nodes transmit their coordinates to the gateway once every 0.5 seconds, and the optimization calculations are also repeated with this frequency. The simulations are run for a total of 5000 seconds.

In Figure 2, simulation data was collected for three different scenarios: a gateway that is placed statically at the center of its domain; a gateway that is moving according to a random waypoint model; and finally, a gateway, the locus of whose trajectory is being updated using our optimization method.

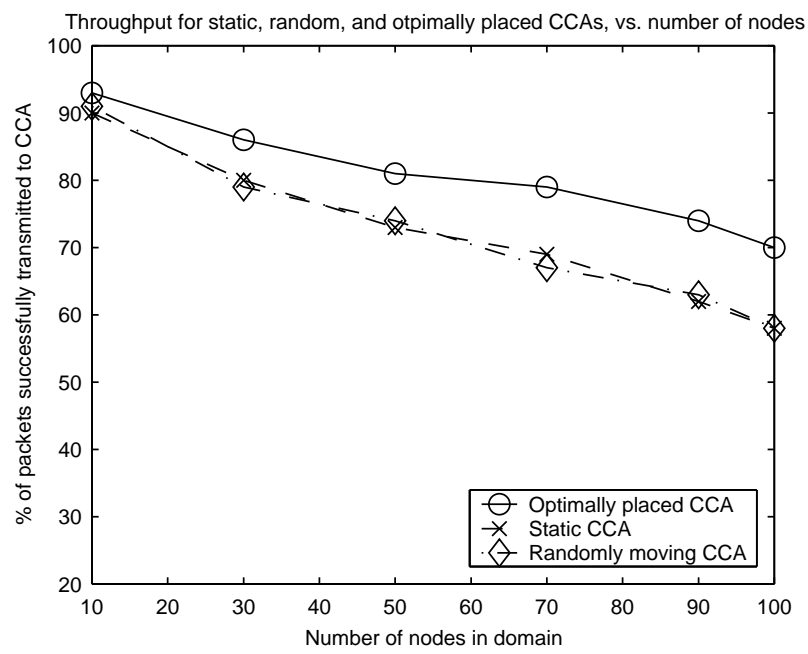

Fig. 2. Comparison of network throughput with optimally placed gateway versus statically placed or randomly moving gateway.

Note that when our algorithm is implemented, the improvement in throughput is as high as $10 \%$ per domain (ignoring inter-domain interference effects), and hence can be very significant for the network as whole when there are multiple gateway domains.

The advantage of the dynamic gateway placement technique is much more evident when we increase the area covered by the blockages (Figure 3).

In Figure 3, initially, with very few blockages in the domain, the number of packets that are successfully transmitted to the gateway is roughly the same for the two cases, i.e., the case in which the gateway is placed statically at the center of the domain, and the case in which the gateway is optimally positioned. However, as the numbers of blockages 


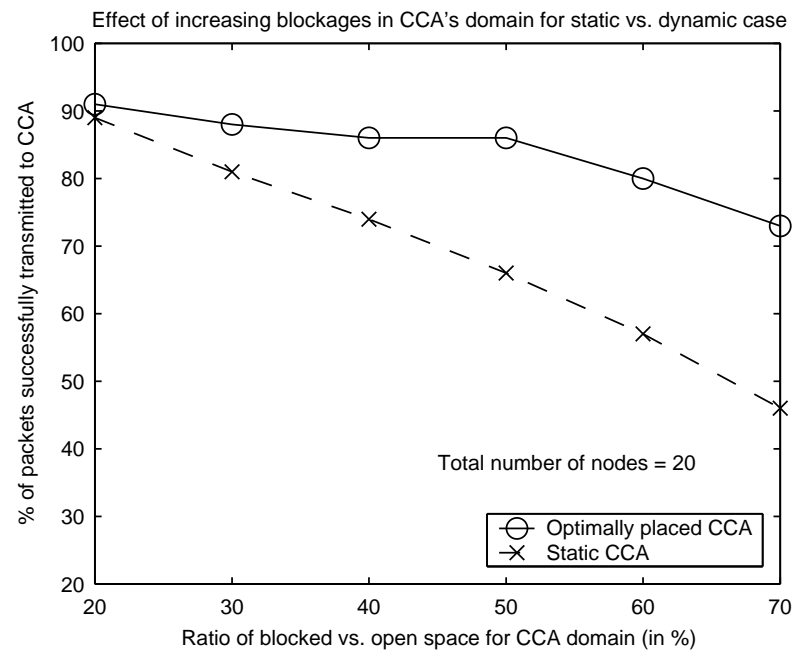

Fig. 3. Effect of blockages on the performance of the static vs. optimally placed gateways.

are increased causing the area covered by the blockages to increase, the open space in the domain decreases as a result, and the throughput drops dramatically if the gateway is statically placed. When the gateway is optimally placed, we noticed the improvement in throughput to be as high as $60 \%$.

To test the performance of the network when the cost function is altered to incorporate different priorities for different nodes, 100 nodes were deployed and the load generated by each node was progressively increased from $10 \%$ to $90 \%$. Note that a node's priority depends on the priority of the packets that it generates at the given time. This is dynamic, akin to the offered load, and changes with time. For a specific value of the offered load, half the nodes (chosen randomly) generated high priority traffic $\left(\eta\left(\rho_{i}, \tau_{i}\right)=\tau_{i}=10\right)$, whereas the remaining nodes generated low priority data traffic $\left(\eta\left(\rho_{i}, \tau_{i}\right)=\tau_{i}=1\right)$. For each of these cases, the average message latency was measured while using the gateway trajectory update algorithm with, and without, the priority class as a parameter in the cost function. The results are depicted in Figure 4. The gateway now favors the nodes generating the higher priority traffic. These packets are delivered more efficiently-directly instead of via multiple hops-and with improved latency. Since the system capacity is fixed, the price that is paid, however, is that the lower priority traffic suffers increased latency and reduced throughput. We also note that, as expected, when the cost function in the optimization formulation does not take packet priorities into account, but considers only the position and offered load, there is no significant difference in the latency incurred by the different classes of traffic.

\section{CONCLUSIONS AND FUTURE WORK}

To support scalability in ad hoc networks, a range extension network can be used. This consists of airborne nodes interfaced with the ad hoc network via gateways that relay data

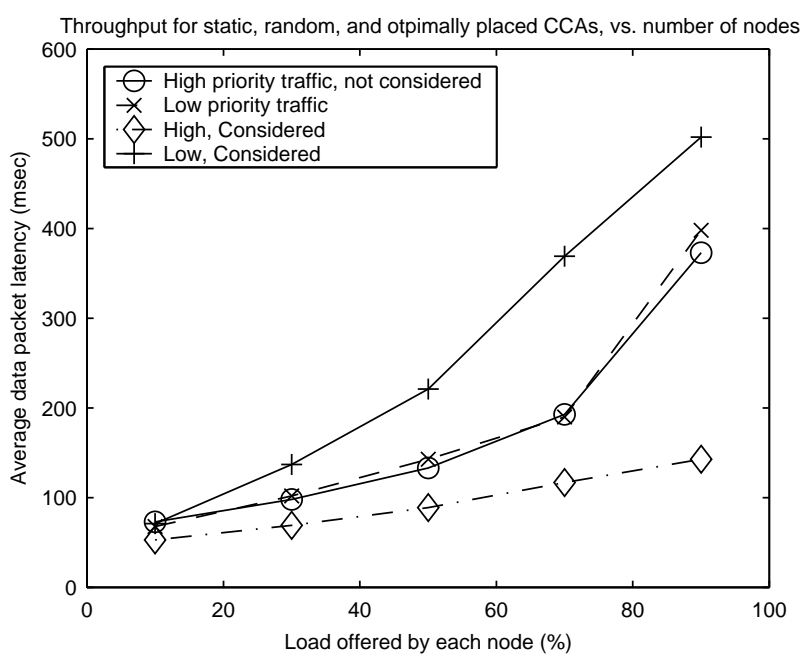

Fig. 4. Effect of including the effect of priority in the optimization cost function.

traffic from/to the ad hoc group to/from the range extension network. The focus of this work has been to determine where these mobile gateways ought to be placed relative to the ad hoc group of nodes, such that certain network performance metrics are optimized. This objective can be formulated as a set of convex optimization problems. By means of suitable modifications, we simplify these convex formulations such that they can be very efficiently solved by numerical methods. We enforce the gateway to follow the computed optimal trajectory, and evaluate the achieved improvements in performance in terms of throughput and latency.

Simulation results indicate that the network throughput improves by about $10-15 \%$ per gateway domain if the gateway moves in accordance with the optimally computed trajectory, as opposed to being static or moving randomly. A similar improvement is seen in terms of a reduction in latency that the data packets experience. The cost function in the optimization formulation can also be appropriately modified to support better performance for prioritized traffic. We also showed that the operations required, in order to thus define an optimal trajectory for the mobile gateways, can be efficiently performed with current hardware technologies and with little additional overhead.

One particular extension of interest is to consider the case wherein multiple gateways are present in a particular domain, where the nodes have the ability to choose different gateways for relaying their inter-domain traffic. This structure can be exploited to balance the loads in different domains, thereby improving performance further. This is currently being investigated.

\section{ACKNOWLEDGEMENTS}

This work was supported by Defense Advanced Research Projects Agency (DARPA) Contract No. N00014-99-C-0322. 
The authors also wish to thank Gregory Pottie (UCLA), and Yongguang Zhang (HRL), for valuable discussions and input.

\section{REFERENCES}

[1] Z. Haas, et. al., "Guest Editorial on Wireless Ad Hoc Networks", IEEE Journal on Selected Areas in Communications, Vol. 17 No. 8,August 1999.

[2] B. M. Leiner, "Goals and challenges of the DARPA GloMo program (global mobile information systems)," IEEE Personal Communications Magazine, Vol. 3, No. 6, pp. 34-43, Dec. 1996.

[3] E. Royer, C.-K. Toh, "A Review of Current Routing Protocols for Ad Hoc Wireless Networks", IEEE Personal Communications Magazine, pp. 46-55, April 1999.

[4] Y. Ko, N. Vaidya, "Using Location Information in Wireless Ad Hoc Networks", Proceedings of the 49th IEEE Vehicular Technology Conference, Vol. 3, pp. 19521956, 1999.

[5] G. Karumanchi, et. al., "Information Dissemination in Partitionable Mobile Ad Hoc Networks", Proceedings of the 18th IEEE Symposium on Reliable Distributed Systems, pp. 4-13, 1999.

[6] K. Amouris, et. al., "A Position-Based Multi-Zone Routing Protocol for Wide Area Mobile Ad Hoc Networks", Proceedings of the 49th IEEE Vehicular Technology Conference, Vol. 2, pp. 1365-1369, 1999.

[7] IEEE Standards Committee, "Medium Access Control (MAC) and Physical (PHY) Specifications," IEEE P802.11/D10, Jan. 1999.

[8] C. E. Perkins and E. Royer, "Ad-Hoc On-Demand Distance Vector Routing”, IEEE Workshop on Mobile Computing Systems and Applications, pp. 90-100, February 1999.

[9] C. E. Perkins and P. Bhagwat, "Highly Dynamic Destination-Sequenced Distance-Vector Routing (DSDV) for Mobile Computers", Computer Communication Review, pp. 234-44, Oct. 1994.

[10] D. Bersimas, J. Tsitsiklis, Introduction to Linear Optimization, Athena Scientific, Massachusetts, 1997.

[11] J. Raju, J. J. Garcia-Luna-Aceves, "A comparison of ondemand and table driven routing for ad-hoc wireless networks," IEEE International Conference on Communications (ICC2000), Vol. 3, pp. 1702-1706, 2000.

[12] ns-2 Network Simulator, USC ISI, http://www.isi.edu/nsnam/ns.

[13] http://www.jpl.nasa.gov

[14] http://www.intel.com

[15] http://www.cs.anl.gov 